\title{
Phase separation tunes signal transduction
}

Biomolecular condensates are membraneless cellular subcompartments that often form through liquid-liquid phase separation driven by intermolecular multivalent interactions. Evidence from recent studies implicates liquid-liquid phase separation in processes as diverse as gene regulation, autophagy and membrane receptor signalling, but exactly how the formation of biomolecular condensates regulates these processes is not understood. Two studies published in Science now demonstrate that $2 \mathrm{D}$ biomolecular condensates of membrane-associated signalling complexes increase the residence of signalling effectors within these complexes at the membrane (membrane dwell time), thereby regulating their activity.

Huang et al. investigated the dynamics of RAS activation by its guanine exchange factor SOS. In this pathway, activation of membrane signalling receptors promotes the phosphorylation of integral membrane adaptor protein LAT, leading to the recruitment of soluble adaptor proteins, such

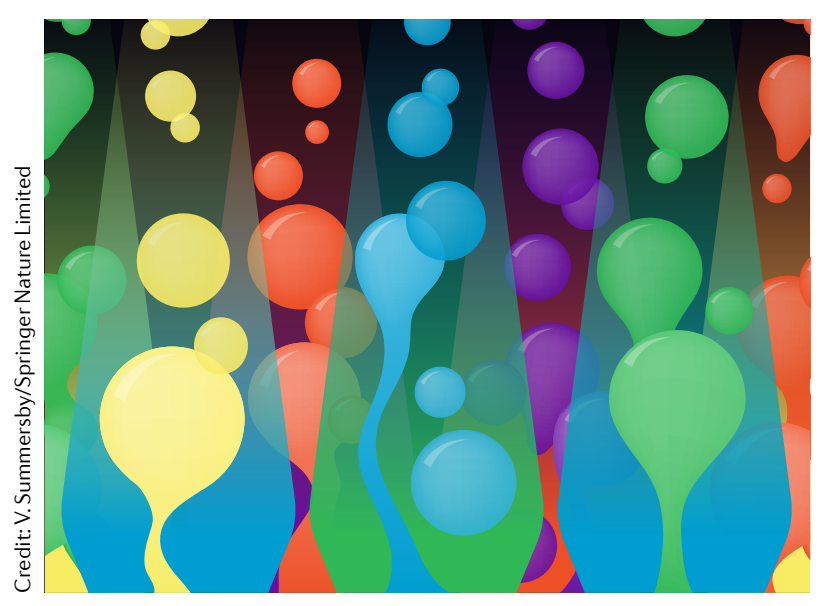

as GRB2. This is followed by the binding and activation of effector molecules, including SOS, for which interaction with the membrane relieves autoinhibition. LAT-GRB2-SOS form $2 \mathrm{D}$ biomolecular condensates at the membrane that increase membrane dwell time of SOS. To understand whether and how this increased membrane dwell time affects RAS-mediated signalling, the authors designed an in vitro reconstitution assay to measure the dynamics of membrane recruitment of single SOS molecules and RAS activation kinetics. The data, together with kinetic models of SOS activation, indicated that membrane-associated SOS transitions through intermediate states before it becomes fully active. This multistep activation process suggests that increased membrane dwell time of SOS might increase the chances of reaching full activation. Indeed, even a small increase in the time that SOS resides in condensates at the membrane (from an average of $\sim 4 \mathrm{~s}$ to $6 \mathrm{~s}$ ) enhanced RAS activation fourfold. This stepwise activation mode is reminiscent of a classical mechanism known as kinetic proofreading, which involves reaction intermediates that increase substrate specificity and reduce errors of biochemical reactions at the expense of increased reaction times required to complete all intermediate steps. The authors propose that a kinetic proofreading mechanism operating during SOS activation at the membrane ensures that RAS activation occurs only in the presence of signal-induced LAT-GRB2-SOS condensates, which have the ability to increase SOS membrane dwell time. These mechanisms would collectively block nonspecific RAS activation, ensuring signalling specificity.
Case et al. studied local F-actin assembly at membrane-associated $2 \mathrm{D}$ biomolecular condensates, formation of which relies on interactions analogous to those in the LAT-GRB2-SOS system, despite involving a distinct set of proteins: phosphorylated Nephrin (membrane receptor), NCK (adaptor protein) and N-WASP (NCK-interacting protein and the activator of actin nucleation factor Arp2/3 complex). The authors observed that rates of Arp2/3-driven actin polymerization were higher inside condensates than outside condensates, which was attributed to increased N-WASP activity within condensates. Similar to SOS, this upregulation of N-WASP activity correlated with increased membrane dwell time of N-WASP (as well as Arp2/3) within condensates. Importantly, N-WASP membrane dwell time was sensitive to NCK concentration: as NCK concentration increased, dwell time initially extended, but then decreased beyond a saturation point. This saturation point probably reflects the exhaustion of phosphorylated sites on Nephrin with subsequent decrease in multivalent connectivity, whereby N-WASP becomes decorated with free NCK (not bound to phospho-Nephrin). Thus, the relative stoichiometry of condensate components - by modulating multivalent interactions within the condensate - controls their dwell times within signalling complexes and consequently fine-tunes their activity.

Because multivalent interactions and multistep reaction cascades are common in signal transduction pathways, these principles of signalling regulation by biomolecular condensates are likely general.

Paulina Strzyz

ORIGINAL ARTICLES Case, L. B. et al. Stoichiometry controls activity of phaseseparated clusters of actin signaling proteins. Science 363, 1093-1097 (2019) | Huang, W. Y. C. et al. A molecular assembly phase transition and kinetic proofreading modulate Ras activation by SOS. Science 363, 1098-1103 (2019) FURTHER READING Banani, S. F. et al. Biomolecular condensates: organizers of cellular biochemistry. Nat.Rev. Mol.Cell Biol. 18, 285-298 (2017) 